\title{
Laser interstitial thermal therapy for radiation necrosis / adverse inflammatory responses to srs - case report and literature review
}

\begin{abstract}
Brain metastases are the most common type of intracranial tumor, and confer a dismal prognosis. Morbidity in these patients arises not only from tumor burden but from treatmentrelated toxicities. Among the most significant of these is radiation necrosis, a common sequelae of stereotactic radiosurgery that may cause neurologic impairment through mass effect or steroid-refractory edema. Truly, "radiation necrosis" is a misnomer, as the process is more accurately an adverse inflammatory response post-stereotactic radiotherapy (AIRS). Because it is impossible to reliably distinguish AIRS from tumor recurrence without a biopsy, and because high-dose steroids are poorly tolerated, management of AIRS poses a significant therapeutic challenge. Laser interstitial thermal therapy (LITT) is an emerging minimally-invasive treatment modality which uses hyperthermia to ablate intracranial pathologic tissues. Here, we describe the use of LITT in a patient with steroid-refractory AIRS following radiosurgery for a right supplementary motor area metastatic focus, and provide a brief review of the utility of LITT in patients with AIRS.
\end{abstract}

Volume 7 Issue 2 - 2017

\author{
Vadim Tsvankin, ${ }^{1,2}$ Elizabeth Howell, ${ }^{1,2}$ Peter \\ Fecci $^{1,3}$ \\ 'Department of Neurosurgery, Duke University Medical Center, \\ USA \\ ${ }^{2}$ The Preston Robert Tisch Brain Tumor Center, Duke University \\ Medical Center, USA \\ ${ }^{3}$ Department of Pathology, Duke University Medical Center, \\ USA
}

Correspondence: Peter Fecci, Duke Brain Tumor Immunotherapy Program, Department of Neurosurgery, Duke University Medical Center, Box 35, Durham, NC 277I, USA, Email peter.fecci@duke.edu

Received: June 20, 2017 | Published: July 05, 2017

\section{Introduction}

Brain metastases are the most common type of intracranial tumor, ${ }^{1-4}$ and confer a dismal prognosis; despite aggressive secondary and even tertiary resections, stereotactic radiosurgery, high-dose external beam radiotherapy, and multi-mechanistic chemotherapy delivered at toxic doses, median survival ranges from 2 to 25 months. ${ }^{5}$ The morbidity of intracranial tumors is substantial, and arises not only from neurological deficits associated with direct brain compression or invasion, but also secondary to systemic and local treatment modalities. In particular, radiation necrosis, a common sequelae of stereotactic radiosurgery (SRS), results in difficult-to-control mass effect and perilesional edema, severely limiting a patient's ability to function and reducing quality of life. ${ }^{6-8}$ Truly, "radiation necrosis" is a misnomer, as the process is more accurately an adverse inflammatory response poststereotactic radiotherapy (AIRS). The mainstay of treatment is highdose corticosteroids, which themselves generate a litany of poorlytolerated symptoms, including hyperglycemia, elevated infection risk, impaired wound healing, osteopenia and suppressed adrenal function. ${ }^{9}$ Alternative strategies such as therapeutic anticoagulation, bevacizumab, ${ }^{10}$ hyperbaric oxygen, ${ }^{11}$ and even resection ${ }^{7}$ have been attempted with limited success, and AIRS remains a substantial therapeutic challenge.

Treatment is further confounded by the clinical and radiographic impossibility of distinguishing AIRS from the most feared eventuality in patients with previously-treated brain tumors: recurrence. ${ }^{12-15}$ Both possibilities are commonly encountered - AIRS typically manifests between three months and several years following SRS, ${ }^{14}$ with an incidence is as high as $50 \%$ at treatment doses between 16 and $22 \mathrm{~Gy}$ to the tumor margin; ${ }^{16-18}$ local recurrence after SRS is as high as $18 \%$ at one-year follow-up ${ }^{19}$ and increases to $31 \%$ at two years. ${ }^{20}$ Lesions showing radiographic enlargement within nine months of SRS have a roughly equal probability of demonstrating AIRS or disease progression on biopsy; lesions enlarging more than nine months post-SRS are much more likely to be AIRS. ${ }^{21}$ The need to distinguish the two entities arises from differential treatment - recurrent tumors necessitate urgent reinstatement of systemic therapy, local cytoreduction (in the form of salvage radiation or resection), or both, while AIRS has been historically managed symptomatically with steroids. ${ }^{22}$ Therefore, suspicion of either entity compels histologic diagnosis. The ideal treatment strategy for both post-SRS contingencies would simultaneously distinguish the two and offer the possibility of immediate treatment, regardless of diagnosis.

To that end, laser interstitial thermal therapy (LITT) is an emerging treatment modality that may address these limitations. Initially developed as an alternative to surgery, LITT offers minimally-invasive cytoreduction in patients with significant comorbidities or difficult-toaccess intracranial lesions. ${ }^{23}$ Initial results have shown the feasibility of LITT for a variety of intracranial pathologies, including new and recurrent metastatic tumors, ${ }^{15,24,25}$ post-treatment edema ${ }^{26}$ radiation necrosis, ${ }^{27}$ epileptogenic foci, ${ }^{28}$ and primary brain tumors such as glioblastoma. ${ }^{29}$ Briefly, a neurosurgeon uses a stereotactically-guided laser to apply low-voltage energy to an intracranial lesion, generating hyperthermia; the process is guided with real-time MRI thermography in a manner that enables the surgeon to direct heat application across the lesion volume, resulting in controlled cell death. ${ }^{30}$ In contrast to open surgery, LITT can be performed through the same basic operating platform as a biopsy, allowing the neurosurgeon to avoid the risk of performing a craniotomy. The incision is less than $1 \mathrm{~cm}$ long; at our institution, patients do not require ICU admission postoperatively, and most are discharged either the same day or the following morning. The result is a minimally-morbid diagnostic and treatment option for patients with previously-treated intracranial tumors. Here, we offer an illustrative case and brief review of LITT application in the setting of AIRS.

\section{Case report}

The patient is a 66 year old right-handed female with a history of lung adenocarcinoma, initially diagnosed in January of 2014 when she presented with cough and hemoptysis. Surveillance imaging demonstrated a solitary $1 \mathrm{~cm}$ metastasis of the right frontal lobe, and 
the patient was treated with Cyber Knife on 3/5/2014. She underwent concurrent chemoradiation with carboplatin/paclitaxel and 60Gy radiation to the right upper lung, mediastinum and $\mathrm{SCV}$ region (completed 4/16/2014), followed by an additional two cycles of chemotherapy (completed 5/20/2014). She was subsequently placed on erlotinib due to EGFR mutation, then switched to afatenib in September 2014.

In July 2015 the patient noted a sense of imbalance and left hand weakness; an MRI demonstrated enlargement of the previouslytreated brain metastasis at the posterior margin of the right superior frontal gyrus, with significant associated edema (Figure 1). She was started on dexamethasone, with some symptomatic improvement. Given her continued weakness, however, a plan was made for the patient to undergo stereotactic biopsy with possible subsequent LITT.

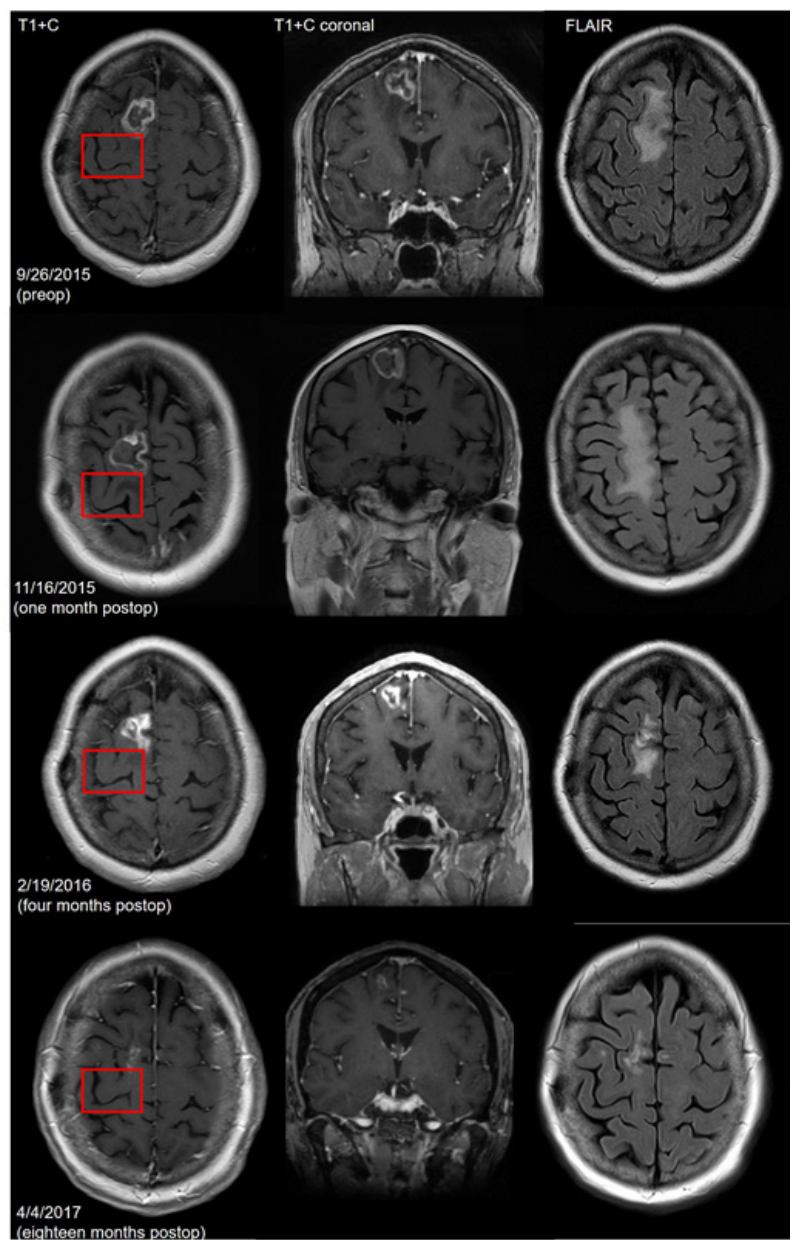

Figure I MRI sequences (axial TI-weighted with contrast, left; coronal TI-weighted with contrast, middle; FLAIR, right) throughout the patient's treatment course. Top row: two weeks prior to treatment; second row: onemonth followup; third row: four-month follow up; bottom row: eighteenmonth followup. Red box denotes hand motor cortex.

On $10 / 12 / 2015$ the patient was electively taken to the operating room. Using frameless stereotaxy, a biopsy was performed; analysis of the frozen specimen was consistent with radiation necrosis. The decision was made to proceed with LITT. Using the Monteris operating platform and software, and under real-time MRI thermographic guidance, laser ablation proceeded in multiple rounds in 360 degrees according to standard protocols. The laser probe was advanced from superficial to deep areas, with $100 \%$ of the lesion covered by "blue line," and particular attention paid to the posterior border abutting eloquent motor cortex. After the uncomplicated procedure, the patient was admitted to the neurosurgery service for overnight observation. She was discharged in stable condition the following morning, with trace weakness in her left hand. Final pathology from the operating room was consistent with radiation necrosis.

At one-month follow-up on 11/16/2017, the patient had regained near-full strength in her left hand, and had otherwise remained clinically well. Her dexamethasone dose was tapered slowly, and she was weaned entirely off roughly nine weeks postoperatively. Her brain MRI (Figure 1) initially demonstrated an enlarged, thinned rim of enhancement of the right frontal lesion, with increased surrounding edema; these were expected changes. The lesion contracted over time (Figure 1), and the most recent MRI (4/4/2017) shows a thin rind of enhancing scar with minimal surrounding FLAIR signal. She is neurologically intact, and otherwise clinically stable.

\section{Discussion}

AIRS is perhaps the most significant late toxicity associated with SRS, causing neurologic symptoms in up to one third of treated patients. ${ }^{16,18,31,32}$ The underlying inflammatory mechanism is thought to result from dysfunctional astrocytes and microglia surrounding the central necrotic zone of the treated lesion. ${ }^{7}$ These perinecrotic cells secrete inflammatory cytokines such as VEGF, TNF- $\alpha$, and ICAM1 , resulting in blood-brain barrier breakdown and angiogenesis; the resultant contrast-enhancing rim apparent on imaging makes it impossible to distinguish from progressive tumor. ${ }^{7,27,32-34}$ The resulting edema promotes perilesional hypoxia, driving HIF-1 $\alpha$ expression $^{35}$ and stimulating a VEGF feedback loop, perpetuating a cycle of edema. Surgical resection of the perinecrotic contrast-enhancing zone in AIRS has been shown to often result in radiographic and clinical improvement; ${ }^{36}$ in the case of LITT, the goal of treatment is transformation of the perinecrotic zone into an area of inactive, factordepleted coagulative necrosis. ${ }^{33}$

In this case, surgical extirpation would have alleviated the mass effect directly, with the additional benefit of offering a histologic diagnosis. However, the lesion was in the superior frontal gyrus, within the supplemental motor area and abutting motor cortex; the associated edema can be seen infiltrating hand motor cortex (Fig. 1, FLAIR sequences). Radiation often confounds reliable intraoperative distinction between lesion and surrounding radiated white matter, making radiated lesions in eloquent areas at times a surgical challenge. The surgical approach thus carried significant risk of postoperative motor deficits. In contrast, the LITT approach offered the same diagnostic and therapeutic potential, with a considerably favorable risk profile and the ability to bring treatment lines to easily distinguished lesion borders on imaging. The first report of LITT for AIRS occurred under similar circumstances, with Rahmathulla et al., ${ }^{37}$ describing a 74-year-old with steroid-refractory edema following SRS for intracranial non-small cell lung cancer metastasis. The lesion's deep location within the centrum semiovale precluded surgery; following biopsy and LITT, the patient was discharged within 48 hours, was weaned off steroids, and demonstrated radiographic improvement at seven-week follow-up. Fabiano \& Alberico ${ }^{26}$ published a similar case report in 2014, describing a 64-year-old patient treated with SRS for a lung adenocarcinoma metastasis in the right external capsule. The patient similarly developed medically-refractory edema, requiring high-dose steroids to remain ambulatory. Following LITT, he was weaned off steroids within two weeks, and maintained his strength during the follow-up period. Our experience is consistent with this clinical course, and suggests that the greatest potential 
benefit LITT may offer to patients with AIRS is the possibility of weaning off steroids; however, we have seen significant variation in how aggressively we are able to taper steroids, from several weeks to several months. In a small series of patients with histologicallyproven radiation necrosis treated with LITT, ${ }^{15}$ Torres-Reveron et al., ${ }^{15}$ described all patients as tolerating the procedure well enough to be discharged within 48 hours; four had durable improvement of neurological symptoms, and all were weaned off steroids within two months. Other authors have published similar results..$^{24,37,38}$

Importantly, we and others have concluded that short-term followup MRI must be interpreted carefully when assessing treatment effect. For two weeks to two months following LITT, lesions tend to increase in size, but develop a thinner "eggshell" rim of enhancement. The appearance is radiographically similar to pseudoprogression, a possible cause for undue concern among radiologists and oncologists unfamiliar with the procedure reading these interpretations. Rao et al., ${ }^{27}$ noted that overall, "the majority of lesions returned to their preablation size in an average of 16 weeks." Carpentier et al., ${ }^{24}$ also noted a similar radiographic increase in lesion volume up to two weeks, followed by steady regression. A number of groups have attempted a formal assessment of MRI patterns following LITT. ${ }^{39}$ Initial images are frequently characterized by decreased contrast enhancement within the center of the lesion; this is surrounded peripherally with a contrastenhancing margin, which confers an "eggshell-like" appearance to the lesions. ${ }^{33}$ Beyond the peripheral zone, a margin dose of edema is seen, hyperintense on T2-weighted images, which can enlarge to 1.5 times its original size and remain enlarged for up to forty days. ${ }^{33,40-42}$ This is generally followed by resorption of the necrotic center, a process extending over several months. ${ }^{33,40}$ Of note, groups have found no correlation between preoperative tumor size, radiographic edema, and probability of symptomatic postprocedural edema ${ }^{24,27,43}$ or steroid dependence. ${ }^{27}$ We hypothesize that steroid-dependence is mostly location-dependent, and varies by proximity to eloquent areas. These data suggest caution in the interpretation of early postoperative radiographic studies, as well as vigilance in patients with increased enhancement volume beyond four to 6 months postoperatively. In our hands, it is not uncommon for a lesion to resemble its original radiographic appearance for up to 6 months postoperatively before beginning to retract into a scar-like appearance on imaging (Figure 2).

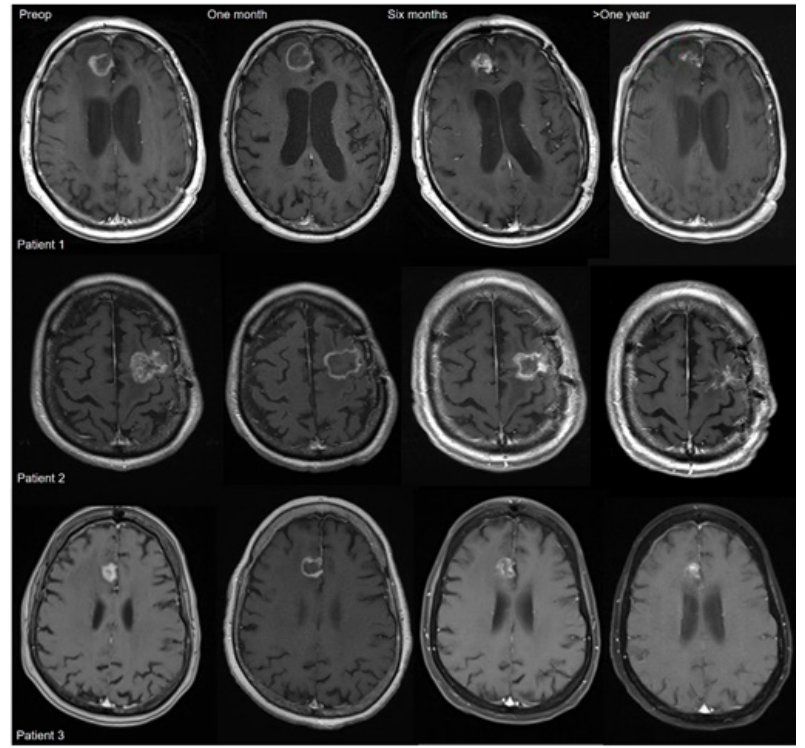

Figure 2 Serial images (TI-weighted MRI with contrast), taken preoperatively, then at one-month, six-month, and long-term (over one year) followup, of three representative patients treated with LITT.
This study adds to the growing body of literature suggesting a role for LITT in the treatment of patients with AIRS, and refocuses the reader on the proper etiology of these lesions: treatable inflammation. Larger studies with longer follow-up periods are necessary to address factors such as patient quality of life, probability of steroid dependence, and neurological outcomes; these will guide and better define the patients that can most benefit from this therapy.

\section{Acknowledgments}

None.

\section{Conflicts of interest}

None.

\section{Funding}

None.

\section{References}

1. Sperduto PW, Chao ST, Sneed PK, et al. Diagnosis-specific prognostic factors, indexes, and treatment outcomes for patients with newly diagnosed brain metastases: a multi-institutional analysis of 4,259 patients. Int J Radiat Oncol Biol Phys. 2010;77(3):655-661.

2. Zimm S, Wampler GL, Stablein D, et al. Intracerebral metastases in solid-tumor patients: natural history and results of treatment. Cancer. $1981 ; 48(2): 384-394$

3. Schouten LJ, Rutten J, Huveneers HA, et al. Incidence of brain metastases in a cohort of patients with carcinoma of the breast, colon, kidney, and lung and melanoma. Cancer. 2002;94(10):2698-2705.

4. Ostrom QT, Gittleman H, Fulop J, et al. CBTRUS Statistical Report: Primary Brain and Central Nervous System Tumors Diagnosed in the United States in 2008-2012. Neuro Oncol 17 Suppl.2015;4:iv1-iv62.

5. Sperduto PW, Kased N, Roberge D, et al. Effect of tumor subtype on survival and the graded prognostic assessment for patients with breast cancer and brain metastases. Int J Radiat Oncol Biol Phys. 2012;82(5):2111-2117.

6. Miyatake S, Nonoguchi N, Furuse M, et al. Pathophysiology, diagnosis, and treatment of radiation necrosis in the brain. Neurol Med Chir (Tokyo). 2015;55(1):50-59.

7. Rahmathulla G, Marko NF, Weil RJ. Cerebral radiation necrosis: a review of the pathobiology, diagnosis and management considerations. $J$ Clin Neurosci. 2013;20(4):485-502.

8. Lubelski D, Abdullah KG, Weil RJ, et al. Bevacizumab for radiation necrosis following treatment of high grade glioma: a systematic review of the literature. J Neurooncol. 2013;115(3):317-322.

9. Armstrong TS, Ying Y, Wu J, et al. The relationship between corticosteroids and symptoms in patients with primary brain tumors: utility of the Dexamethasone Symptom Questionnaire-Chronic. Neuro Oncol. 2015;17(8):1114-1120.

10. Torcuator R, Zuniga R, Mohan YS, et al. Initial experience with bevacizumab treatment for biopsy confirmed cerebral radiation necrosis. J Neurooncol. 2009;94(1):63-68.

11. Kohshi K, Imada H, Nomoto S, et al. Successful treatment of radiationinduced brain necrosis by hyperbaric oxygen therapy. J Neurol Sci. 2003;209(1-2):115-117.

12. Dooms GC, Hecht S, Brant-Zawadzki M, et al. Brain radiation lesions: MR imaging. Radiology. 1986;158(1):149-155.

13. Tsuruda JS, Kortman KE, Bradley WG, et al. Radiation effects on cerebral white matter: MR evaluation. AJR Am J Roentgenol. 1987;149(1):165-171. 
14. Sharma M, Balasubramanian S, Silva D, et al. Laser interstitial thermal therapy in the management of brain metastasis and radiation necrosis after radiosurgery: An overview. Expert Rev Neurother. 2016;16(2):223-232.

15. Torres-Reveron J, Tomasiewicz HC, Shetty A, et al. Stereotactic laser induced thermotherapy (LITT): a novel treatment for brain lesions regrowing after radiosurgery. $J$ Neurooncol. 2013; 113(3):495-503.

16. Flickinger JC, Lunsford LD, Kondziolka D, et al. Radiosurgery and brain tolerance: an analysis of neurodiagnostic imaging changes after gamma knife radiosurgery for arteriovenous malformations. Int J Radiat Oncol Biol Phys. 1992;23(1):19-26.

17. Voges J, Treuer H, Lehrke R, et al. Risk analysis of LINAC radiosurgery in patients with arteriovenous malformation (AVM). Acta Neurochir Suppl. 1997;68:118-123.

18. Blonigen BJ, Steinmetz RD, Levin L, et al. Irradiated volume as a predictor of brain radionecrosis after linear accelerator stereotactic radiosurgery. Int J Radiat Oncol Biol Phys. 2010;77(4):996-1001.

19. Aoyama H, Shirato H, Tago M, et al. Stereotactic radiosurgery plus whole-brain radiation therapy vs stereotactic radiosurgery alone for treatment of brain metastases: a randomized controlled trial. Jama. 2006;295(21):2483-2491.

20. Kocher M, Soffietti R, Abacioglu U, et al. Adjuvant whole-brain radiotherapy versus observation after radiosurgery or surgical resection of one to three cerebral metastases: results of the EORTC 22952-26001 study. J Clin Oncol. 2011;29(2):134-141.

21. Narloch JL, Farber SH, Sammons S, et al. Biopsy of enlarging lesions after stereotactic radiosurgery for brain metastases frequently reveals radiation necrosis. Neuro Oncol. 2017.

22. Stockham AL, Suh JH, Chao ST, et al. Management of recurrent brain metastasis after radiosurgery. Prog Neurol Surg. 2012;25:273-286.

23. Mohammadi AM, Schroeder JL. Schroeder, Laser interstitial thermal therapy in treatment of brain tumors--the NeuroBlate System. Expert Rev Med Devices. 2014;11(2):109-119.

24. Carpentier A, McNichols RJ, Stafford RJ, et al. Laser thermal therapy: real-time MRI-guided and computer-controlled procedures for metastatic brain tumors. Lasers Surg Med. 2011;43(10):943-950.

25. Hawasli AH, Bagade S, Shimony JS, et al. Magnetic resonance imagingguided focused laser interstitial thermal therapy for intracranial lesions: single-institution series. Neurosurgery. 2013;73(6):1007-1017.

26. Fabiano AJ, Alberico RA. Laser-interstitial thermal therapy for refractory cerebral edema from post-radiosurgery metastasis. World Neurosurg. 2014;81(3-4):652.

27. Rao MS, Hargreaves EL, Khan AJ, et al. Magnetic resonance-guided laser ablation improves local control for postradiosurgery recurrence and/or radiation necrosis. Neurosurgery. 2014;74(6):658-667.

28. Hoppe C, Witt JA, Helmstaedter C, et al. Laser interstitial thermotherapy (LiTT) in epilepsy surgery. Seizure. 2017;48:45-52.
29. Thomas JG, Rao G, Kew Y, et al. Laser interstitial thermal therapy for newly diagnosed and recurrent glioblastoma. Neurosurg Focus. 2016;41(4):E12

30. Hawasli AH, Kim AH, Dunn GP, et al. Stereotactic laser ablation of high-grade gliomas. Neurosurg Focus. 2014;37(6):E1.

31. Frazier JL, Batra S, Kapor S, et al. Stereotactic radiosurgery in the management of brain metastases: an institutional retrospective analysis of survival. Int J Radiat Oncol Biol Phys. 2010; 76(5):1486-1492.

32. Williams BJ, Suki D, Fox BD, et al. Stereotactic radiosurgery for metastatic brain tumors: a comprehensive review of complications. $J$ Neurosurg. 2009;111(3):439-448.

33. Rahmathulla G, Recinos PF, Kamian K, et al. MRI-guided laser interstitial thermal therapy in neuro-oncology: a review of its current clinical applications. Oncology. 2014;87(2):67-82.

34. Soussain C, Ricard D, Fike JR, et al. CNS complications of radiotherapy and chemotherapy. Lancet. 2009;374(9701):1639-1651.

35. Nordal RA, Nagy A, Pintilie M, et al. Hypoxia and hypoxia-inducible factor-1 target genes in central nervous system radiation injury: a role for vascular endothelial growth factor. Clin Cancer Res. 2004;10(10):3342-3353

36. Nonoguchi N, Miyatake S, Fukumoto M, et al. The distribution of vascular endothelial growth factor-producing cells in clinical radiation necrosis of the brain: pathological consideration of their potential roles. J Neurooncol. 2011;105(2):423-431.

37. Rahmathulla G, Recinos PF, Valerio JE, et al. Laser interstitial thermal therapy for focal cerebral radiation necrosis: a case report and literature review. Stereotact Funct Neurosurg. 2012;90(3):192-200.

38. Carpentier A, McNichols RJ, Stafford RJ, et al. Real-time magnetic resonance-guided laser thermal therapy for focal metastatic brain tumors. Neurosurgery. 2008;63(1 Suppl 1):ONS21-28.

39. Missios S, Bekelis K, Barnett GH. Renaissance of laser interstitia thermal ablation. Neurosurg Focus. 2015;38(3):E13.

40. Schwabe B, Kahn T, Harth T, et al. Laser-induced thermal lesions in the human brain: short- and long-term appearance on MRI. J Comput Assist Tomogr. 1997;21(5):818-825.

41. Tracz RA, Wyman DR, Little PB, et al. Comparison of magnetic resonance images and the histopathological findings of lesions induced by interstitial laser photocoagulation in the brain. Lasers Surg Med. $1993 ; 13(1): 45-54$

42. Tracz RA, Wyman DR, Little PB, et al. Magnetic resonance imaging of interstitial laser photocoagulation in brain. Lasers Surg Med. 1992;12(2):165-173.

43. Jethwa PR, Barrese JC, GowdaA, et al. Magnetic resonance thermometryguided laser-induced thermal therapy for intracranial neoplasms: initial experience. Neurosurgery. 2012;71(1 Suppl Operative):133-144. 